\title{
Accuracy and Efficiency in Fixed-Point Neural ODE Solvers
}

\author{
Michael Hopkins \\ michael.hopkins@manchester.ac.uk \\ Steve Furber \\ steve.furber@manchester.ac.uk \\ School of Computer Science, APT Group, University of Manchester, \\ Manchester M13 9PL, U.K.
}

Simulation of neural behavior on digital architectures often requires the solution of ordinary differential equations (ODEs) at each step of the simulation. For some neural models, this is a significant computational burden, so efficiency is important. Accuracy is also relevant because solutions can be sensitive to model parameterization and time step. These issues are emphasized on fixed-point processors like the ARM unit used in the SpiNNaker architecture. Using the Izhikevich neural model as an example, we explore some solution methods, showing how specific techniques can be used to find balanced solutions.

We have investigated a number of important and related issues, such as introducing explicit solver reduction (ESR) for merging an explicit ODE solver and autonomous ODE into one algebraic formula, with benefits for both accuracy and speed; a simple, efficient mechanism for cancelling the cumulative lag in state variables caused by threshold crossing between time steps; an exact result for the membrane potential of the Izhikevich model with the other state variable held fixed. Parametric variations of the Izhikevich neuron show both similarities and differences in terms of algorithms and arithmetic types that perform well, making an overall best solution challenging to identify, but we show that particular cases can be improved significantly using the techniques described.

Using a $1 \mathrm{~ms}$ simulation time step and 32-bit fixed-point arithmetic to promote real-time performance, one of the second-order Runge-Kutta methods looks to be the best compromise; Midpoint for speed or Trapezoid for accuracy. SpiNNaker offers an unusual combination of low energy use and real-time performance, so some compromises on accuracy might be expected. However, with a careful choice of approach, results comparable to those of general-purpose systems should be possible in many realistic cases.

1 Introduction

In computational neuroscience, most neuron models are specified in the form of ordinary differential equations (ODEs) or occasionally partial 
differential equations (PDEs), which are themselves usually reduced to ODEs for efficient solution. The state variables within the equation model the internal state of the neuron at any particular point in time, and the ODE describes its dynamic behavior. The state variables are initialized at the start of the simulation, and then the evolution of the neuron behavior is computed by solving the ODE at each time point, taking into account state variables and input history. There is a voluminous literature on the numerical solution of initial value ODEs going back at least to the work of Euler in 1770 (Bashforth \& Adams, 1883; Runge, 1895; Heun, 1900; Kutta, 1901; Euler, 1913; Gear, 1971; Lambert, 1973, 1991; Hall \& Watt, 1976; Butcher, 2003). A large variety of methods are available, and many trade-offs exist among the three key performance measures of accuracy, stability and efficiency (the last being defined in our context as "time taken per neural update").

This letter aims to assess the options and find practical solutions that are biased toward a particular set of circumstances while, we hope, also highlighting some more general principles and possibilities. In order to get a balance between breadth and depth of analysis within space constraints, we have chosen to look only at the Izhikevich model with a limited set of variations in parameterization, input behavior, and initial conditions. We hope that some of the ideas and methods will be useful more widely. The set of circumstances we focused on here are

1. 32-bit fixed-point arithmetic

2. Run-time efficiency as a primary motivation

3. The Izhikevich neural model

The first two requirements stem from the need to execute on the ARM968 processor used in the SpiNNaker architecture described in Furber, Galluppi, Temple, and Plana (2014), which does not contain a hardware floatingpoint unit, and the challenging aim of simulating large-scale neural models in real time. The third requirement arises because the Izhikevich model is widely used and provides a large number of realistic neural behaviors while remaining manageable in terms of computational load. The continuous part of the Izhikevich ODE model is defined in Izhikevich (2003) as:

$$
\begin{aligned}
& \frac{d V}{d t}=140+(5+0.04 V) V-U+I, \\
& \frac{d U}{d t}=a(b V-U),
\end{aligned}
$$

where $V$ represents a notional membrane voltage in $\mathrm{mV}, U$ represents a dimensionless membrane recovery variable, $I$ is the input current to the neuron in $\mathrm{nA}$, and $a$ and $b$ are dimensionless parameters that define the dynamic behavior of the recovery variable in terms of timescale and the sensitivity to subthreshold fluctuations in $V$, respectively. A second part of the model 
defines the discontinuity that occurs when $V$ hits the firing cutoff of the neuron,

$$
\begin{aligned}
& V \rightarrow c, \\
& U \rightarrow U+d,
\end{aligned}
$$

$c$ being the postspike reset value for $V$ and $d$ being the postspike offset for $U$. Thirty $\mathrm{mV}$ appears to be the defacto standard cutoff value for this parameterization of the model, and we have also chosen to use it. As Touboul (2009) showed, the solution can be very sensitive to this value, and there is an argument that it is therefore an important fifth parameter. Looking at our results compared to Touboul, we do not appear to suffer from this sensitivity in any case. It's interesting to observe in Figure 1f of Touboul that there seems to be a "clear patch" where bifurcation behavior is much reduced around the $30 \mathrm{mV}$ cutoff value. Perhaps this has contributed to the common choice of this value.

The presence of this discontinuity in the state variables will be seen to have an effect on both the solution methods that are most appropriate and the refinements required to approximate continuous time when the simulation is computed at discrete time points. It should be noted that many current neural models have a similar discontinuity property (usually called integrate-and-fire models). Izhikevich (2003) showed that by choosing $\{a$, $b, c, d\}$ appropriately, one can reproduce many realistic types of neural behavior. Other studies that look at the options for numerical solution of this type of equation are Humphries and Gurney (2007) and Touboul (2010).

In the following sections, we show that in the context being considered, some ODE solution methods are more effective than others in terms of their balance between efficiency and accuracy and that techniques can be used to improve solutions by appropriately taking into account the specific nature of the SpiNNaker simulation system and arithmetic hardware.

\section{Classes of ODE Methodologies and Examples Chosen}

A convenient way to consider the variety of methods available for solving initial value ODEs is to categorize them according to the nature of the algorithm. A first consideration is explicit versus implicit. Implicit solvers require the solution of a set of inherently nonlinear equations at each time point, usually via iterative algorithms-though direct solutions using linearized approximations are possible in some cases. They provide desirable properties in terms of improved stability when encountering the stiff numerical behavior that can occur when solving neural ODEs. Stiffness in the context of ODEs is explained in Butcher (2003): "These systems are characterised by very high stability, which can turn into very high instability when approximated by standard numerical methods" (p. 27). 
This extra numerical stability does not come for free, as it is significantly more expensive computationally and therefore not of interest to us taking into account our aim of real-time computation with limited computational power. In contrast, explicit solvers use values already available or readily computable and then predict the value(s) of the state variable(s) at the next time point using a direct algebraic formula. Explicit solvers vary widely in their stability characteristics.

Another consideration is fixed versus adaptive step size. Fixed step size uses a constant time step for moving the simulation forward, whereas adaptive step size makes decisions on step size based on the nature of the solution at each time point. Adaptive step size provides desirable properties for all of the usual numerical requirements of accuracy, efficiency, and stability and should always be considered if feasible. However, due to key aspects of the simulation of large-scale neural systems on the SpiNNaker architecture, the complexity and overhead of moving away from the underlying fixed time-step behavior of the system as a whole make the global use of adaptive time steps infeasible. In the spirit of practicality, we have therefore worked with fixed time-step behavior in this study (apart from the reference results used for comparison). It is important to keep this in mind when looking at results, as it has an inevitable impact on the accuracy and stability of solutions.

The order of a method expresses how quickly the prediction error improves as step size reduces, but the exact implication of order differs depending on the method being considered. For example, higher order should reduce prediction error in general, but for some methods, stability will increase while for others it will reduce. It needs to be borne in mind that the sophisticated mathematical analysis underlying these results in most cases assumes exact arithmetic. This can make actual behavior quite different from the formal expectations, as highlighted in the following sections.

Butcher (2003) provides a helpful breakdown of the most commonly used numerical algorithms into the following three dimensions: multistep-use previous values of the state variables; multistage-calculate first derivatives at multiple points between the current and next time point; and multiderivative-use higher derivatives calculated at the current time point. Example explicit methods relevant to our requirements are the following (for details see Gear, 1971; Lambert, 1973):

- Multistep: Linear multistep methods-Adams-Bashforth in simplest form

- Multistage: Runge-Kutta

- Multiderivative: Taylor series and Parker-Sochacki

The basic Euler method is the simplest example of all three types and so, in a sense, defines the origin in this 3D space. Also, hybrid methods that combine features of some or all of the types are possible. A detailed discussion of these potentially interesting hybrids is beyond the scope of 
this letter, but some possibilities are mentioned later. An algorithm that does not fit neatly into this scheme is the extrapolation method; the best-known example is the Gragg-Bulirsch-Stoer (see Lambert, 1973; Press, Teukolsky, Vetterling, \& Flannery, 1992; Stewart \& Bair, 2009). This is probably the first choice when very accurate solutions for smoothly behaving systems are required, but it is not recommended when discontinuities in the state variables are expected or when the derivative calculations are nontrivial. We therefore restrict initial consideration to the following explicit, fixed time step solution methods: Linear Multistep, Runge-Kutta, Taylor Series, and Parker-Sochacki.

All are families of methods that provide different orders. For the RungeKutta methods of order 2 and higher, there is an infinite variety of algorithms for each order adjusted by free parameters that define the explicit equations to be calculated. These considerations are expressed in the form of a Butcher tableau, the most comprehensive description of which is given by Butcher (2003). Computationally, the Runge-Kutta methods require one derivative calculation per order per state variable. The Taylor series method is similar to Runge-Kutta in this respect but unique in analytical form for each order, as is Parker-Sochacki. The relevant explicit linear multistep method (Adams-Bashforth) requires only one derivative calculation per state variable independent of order, making it potentially very efficient.

In order to keep the numerical investigation manageable, we further trim the methods being considered. The Parker-Sochacki method uses truncated Picard iteration to compute a Maclaurin series and has already been successfully applied to the Izhikevich model, among others (for details of how these methods fit together, see Stewart \& Bair, 2009). It offers many opportunities for extra sophistication by, for example, automatically adapting to provide a required level of accuracy and naturally expressing power series, rational and transcendental functions using iterative polynomial operations. It certainly deserves further study, but an initial analysis of the computations required makes it clear that it is unlikely to be competitive in terms of efficiency here and so has not been considered further.

The Adams-Bashforth method has great potential in terms of efficiency, but the stability is poor, especially for higher orders, and in the presence of stiff behavior, it is unlikely to converge reliably with realistic time steps. Incorporating it into predictor-corrector algorithms with an Adams-Moulton corrector and the local extrapolation technique sometimes called Milne's method increases stability significantly but also computational load (see Lambert, 1991, for details of this combination and his Figures 4.1 and 4.2 for a before-and-after comparison). Furthermore it requires extra memory to retain past values-the local fast memory that would be required is at a premium in the SpiNNaker chip_and will not cope well with the discontinuities in the state variables without extra administration code and related overhead. 
So the final methods that are evaluated and compared with each other and against a reference solution computed using the sophisticated ODE solver available in the Mathematica environment (Wolfram Research, 2014) are the Runge-Kutta and Taylor series.

\section{Specifics and Implications of Fixed-Point Architecture}

Compared to a standard numerical computation environment where floating-point types and operations are available, working with fixed-point arithmetic and the various constraints that come with it can be a challenge. Historically, fixed-point arithmetic has usually been carried out using handcoded transformations of integer arithmetic (for an example of this in a closely related context, see Jin, Furber, \& Woods, 2008). A benefit of this approach is that custom types can be generated for specific problems, and key arithmetic operations can be optimized for speed, including the use of assembly language where appropriate. However, it makes the code significantly harder to write, read, and maintain and is prone to hard-to-find bugs. It is therefore not considered ideal for large-scale software engineering where ideas and code need to be shared between groups, especially when those groups may contain more neuroscientists, mathematicians, and engineers than computer scientists. Fortunately, the recent draft standard TR18037 ISO/IEC (2008) offers a well-defined solution to this quandary by types and arithmetic operations that use fixed-point arithmetic, while allowing $\mathrm{C}$ code that looks very similar to that using the usual floating-point types and operations and with all the required manipulations carried out automatically by the compiler. This draft standard has been implemented in the GCC development tool chain since the 4.7 release-currently for the ARM target only. As the SpiNNaker architecture is based on ARM, we can transparently apply this approach for our numerical work, and so the rest of this letter works under this assumption unless stated otherwise. Our chosen default type is defined in the ISO draft as accum-a 32-bit signed type with 16 integer bits and 15 fractional bits (s16.15) that allows efficient arithmetic operations on the ARM processor.

With this consideration dealt with, the other important issues are numerical -in particular, overflow, underflow, and quantization/rounding errors. As discussed briefly in the previous section, most standard results on accuracy and stability for ODE solvers are based on exact arithmetic. Even for double precision floating point, we are a long way from this ideal case, and in our target 32-bit architecture using the s16.15 type, other issues will come into play. Work has been done to look specifically at the effect of rounding error on initial value ODEs (in Gill, 1951; Henrici, 1962; Babuška, Práger, \& Vitásek, 1966; Kahan, 1965; Vitásek, 1969; Higham, 1996), but much of the recent work is in the context of floating-point arithmetic, which has 
different properties from fixed point-in particular, the various compensated summation schemes recommended for floating-point algorithms (see, e.g. section 4.3 of Higham, 1996) are of no benefit in fixed-point calculations.

The earliest three references are exceptions, with Gill (1951) providing the detail of an ingenious mechanism for applying the traditional fourthorder Runge-Kutta method in fixed-point arithmetic while minimizing the effect of rounding errors in the calculation. Henrici (1962) provides an analysis and examples of statistical distributions of rounding errors for a number of explicit algorithms. Chapters 2 and 3 of Babuška et al. (1966) provide an analysis of both fixed-point and floating-point errors in relation to ODE solutions with examples. Although details vary a great deal between their examples and methods, a characteristic V-shaped pattern occurs where the error reduces as step size is reduced until the point where roundoff/quantization causes the error to rise again as step size is reduced further. The slopes of this pattern depend on details of the equation and whether floating-point or fixed-point arithmetic is being used.

Though interesting and relevant to some of our results, a thorough mathematical analysis of the difference between the two types of arithmetic is outside the scope of this letter. One thing that has become apparent, however, is that the sensitivity to rounding error is difficult to characterize analytically because the same algorithm can be implemented differently in terms of, for example, the choice and ordering of interim variables, and this in itself can make a significant difference to the accuracy of final results.

Our search for the best algorithm in each case was entirely manual and clearly not provably optimal. Within that context, however, it was relatively thorough, looking at a large number of possible equation factorizations and orderings for variables and operations. Even with this limited approach, significant differences were found between options that were identical if considered in terms of their algebraic definition. We hope this has at least demonstrated the potential of the approach. Work has been done on automating this process by Martel (2009), Darulova et al. (2013), and Gao, Bayliss, and Constantinides (2013), and this would appear to be a potentially fruitful way to proceed. We would add a note of caution to the effect that fixed-point types require very firm prior knowledge in order to turn the process over to an automated method-in particular, strict guarantees that variables within algorithms will not overflow or underflow the representable range in any possible use case-and that these are sometimes more difficult to provide than might at first seem to be the case.

The key numerical details of working with the accum s16.15 type on an ARM target are:

1. Absolute values $<0.000031$ become zero.

2. Absolute values $\geq 65,536$ wrap around (saturated types are available but are much slower).

3. Precision is absolute rather than relative as with floating point. 
4. Division is very slow (due partly to details of the ARM instruction set) and should be avoided.

5. There is no native transcendental function library (though one is being developed in our group).

As might be expected, these points have a direct impact on the algorithms that we are discussing here and will be referred back to when considering implementations and results.

\section{Implementation Details}

The system language for SpiNNaker is ANSI C, and so this is the language used for the implementation. An API was developed that provides a general but also efficient interface between the neural model API and the ODE solvers that might be of interest. A plug-in architecture was chosen that provides the ability to test new algorithms while also promoting efficiency in regard to such issues as compiler optimizations, memory access patterns, and the use of registers. After some consideration, we arrived at a structure similar to the one used in chapter 16 of Press et al. (1992) but focused on our particular needs.

The API refers to a generic REAL type, which can be instantiated at compile time to any type of interest-in our case, accum, long accum, float, and double. This allows end-to-end comparison of all models and algorithms with one type definition in a header file.

The ODE solver API includes the ability to internally reduce the solution step size independent of the overall simulation time step. This is a useful mechanism for increasing accuracy for a linear cost in efficiency if required. The solver type is in general sent as a function pointer within the code in order to be changed easily and is therefore ideal for comparative work. However, the price to be paid for this generality is the time taken to dereference the pointer at each call. For production code, this overhead can be reduced, with each successive specialization allowing the compiler to shave off instructions required for the solve routine. This can be specialized further if one knows in advance how many state variables will be in a neuron definition by hard-coding the inner loops in the solver. Some examples of the efficiency gains are given in section 7 .

Another possibility for speed improvement that is orthogonal to the specializations just described is the use of static inline functions. Using this technique, code is defined in the header file rather than the source file similar to the usual use of templates in $\mathrm{C}++$. Although the software package is now rather less clean in terms of build and delivery, this technique allows the compiler further optimization opportunities - in particular, code optimizations across source files that would normally be compiled separately and linked at build time. The efficiency benefits of this approach are also discussed in section 7 . 
An important idea that arose while considering implementation was the following. As in any particular case we are working with a fixed step size, explicit solver and known ODE, the calculations at any given time step can be described algebraically instead of algorithmically, the normal implementation method. This can then be manipulated to, for example, simplify and find common terms, which will reduce the computational overhead and allow the compiler to find more optimizations. We have called this explicit solver reduction (ESR), and it turns out that not only does it enable efficiency gains, but also the reduction chosen can help fixed-point arithmetic retain more precision and avoid overflow and underflow conditions and therefore improve accuracy as well. We give more details in the examples. We call these quasi-closed form solutions as they approximate closed-form solutions. Other software engineering benefits are that the use of static inline functions is no longer required, as all relevant compiler optimizations can now be done in the single source file where the computation is defined and any given method/neuron combination can be delivered pretested.

A broadly similar approach has been proposed recently by Stimberg, Goodman, Benichoux, and Brette (2014). Although we have independently come to the conclusion that there are benefits in combining the ODE and solver into an algebraic equation, the motivation and the methods used are different. For them, the emphasis is on a general mechanism for describing models, easy testing of new ideas, and automatic code generation; for us, it has been seen as an algebraic method of finding the best computational trade-off between accuracy and efficiency, while simultaneously taking into account the particular constraints of fixed-point arithmetic.

Like most other neurally inspired ODEs, the Izhikevich model is autonomous, meaning that the equations make no explicit mention of the time variable on the right-hand side; they are a function of neural parameters and values of the state variables only without time being referenced. This allows Taylor series methods to be implemented algebraically in advance for any number of state variables using an elegant approach described by Alsaker (2009). This means that these solver/neuron combinations can be converted to quasi-closed form solutions directly with all the benefits described previously.

\section{Specifics of Neural Context}

Previous work has used different default time steps, usually either $0.1 \mathrm{~ms}$ or $1 \mathrm{~ms}$. The default on SpiNNaker was chosen as $1 \mathrm{~ms}$ to facilitate biological real-time performance. As a result, most of the comparison work uses this time step unless stated otherwise. Over time, this may change in order to achieve more accuracy for ODE solutions and integration of synapses with short time constants, and so some results will also be shown at $100 \mu$ s for comparison. 
A particular feature of many neural ODEs is the discontinuity present when a neuron state variable hits the cutoff and fires. In the case of the Izhikevich model, both state variables have their values instantaneously changed at this point. This generates two requirements for numerical solutions:

1. A solution method that is not troubled by these discontinuities

2. A mechanism to correct for the threshold-crossing point being between time steps

The first has already been discussed. The second we describe here as time quantization (TQ) and requires some more thought.

Correct handling of TQ should be dealt with by any comprehensive ODE solution code, but nave codes will ignore the issue and treat the cutoff point as the next time step after the threshold has been crossed. It is not hard to see that this will inevitably introduce a cumulative lag into the progress of the state variables. Assuming a random uniform distribution of the crossing point between time steps would imply that this approach accumulates an expected lag of $1 / 2$ time step every time the neuron fires. An improvement is possible, but this requires more processing and so must be done efficiently. The simplest method is to add some time to the first evolution of the state variables after the neuron has fired in order to allow it to catch up the lost time. The obvious value to choose would be the expected value of this loss: $1 / 2$ time step so that the first solve after the firing event receives a time value of $1 \frac{1}{2}$ time steps. This removes the expected bias and provides a maximum error of $1 / 2$ time step, a standard deviation for the jitter introduced into the spike timing of about 0.29 time step, and a mean absolute deviation of $1 / 4$ time step. This simple scheme performs quite well and has low overhead. More sophisticated schemes are available, but the most accurate ones require some form of iteration (e.g., Newton-Raphson in section 3.2 of Stewart and Bair, 2009) and would therefore be too expensive to consider given our challenging real time target.

More accurate direct solutions usually require some form of interpolation for the time progress of the state variables between time steps. This is a promising approach, but interpolation inevitably requires at least one arithmetic division, which is very expensive on the ARM processor, and so a technique is needed that approximates the ideal form of interpolation but uses no divisions. We describe a simpler scheme that is based on trigonometric arguments and requires the assumption of linear behavior between the prethreshold and postthreshold time point. The time step is divided into three sections and, depending on where the crossing point is calculated to have occurred, the midpoint of that section is chosen for the next time step (see Figure 1). This again removes the expected bias and provides a maximum error of $1 / 6$ time step, a jitter standard deviation of approximately 0.096 time step, and a mean absolute deviation of $1 / 12$ time step using an 


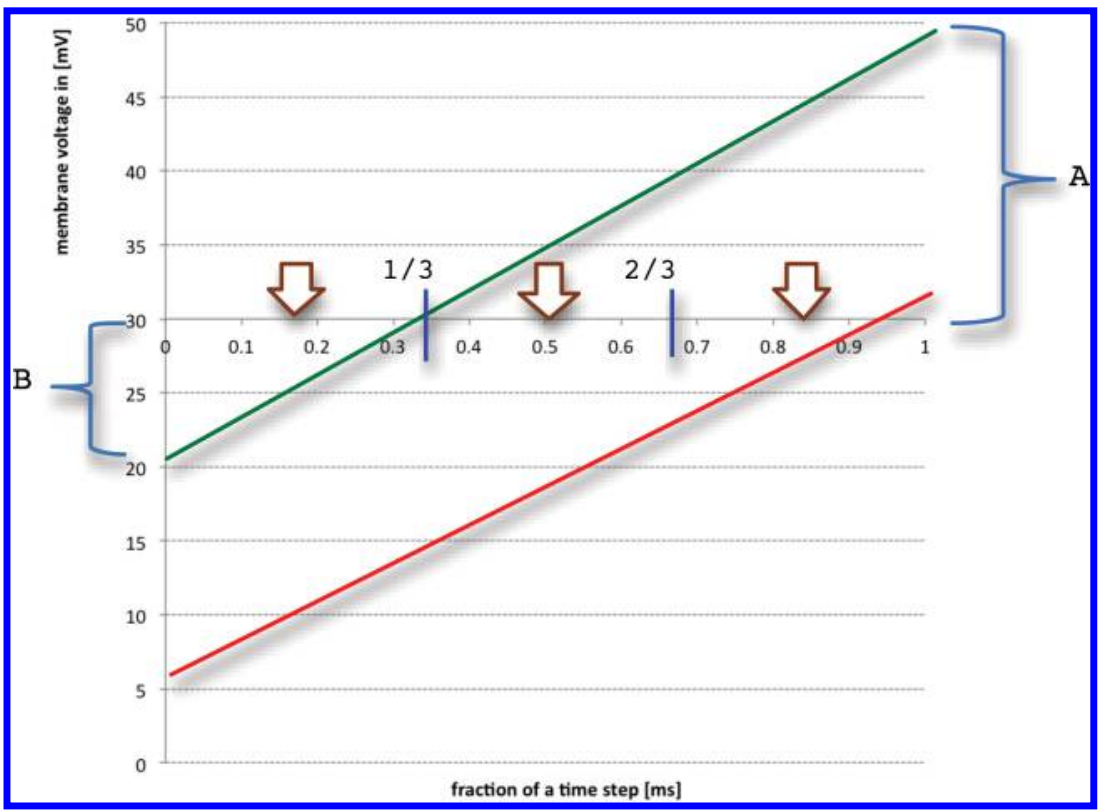

Figure 1: TQ3 correction scheme. The three arrows show the center points of the three sectors, which are expectations over the unknown distribution of threshold crossing time given that it happened in one of the three sectors. So these are used as the best estimate once the sector has been inferred from the pre- and post-threshold state variable value (here in $\mathrm{mV}$ ). The green line shows a state variable trajectory that spikes at the end of the first third and the values of $\mathrm{A}$ and B used in the sample calculation. The red line shows one that spikes in the last third.

expected $^{1}$ computational load of

$$
2 \times \text { subtract }+1.67 \times(\text { multiply }+ \text { compare }),
$$

which is significantly lower than a linear interpolation in its simplest precomputable form:

$$
\text { Divide }+2 \times \text { multiply }+2 \times \text { subtract }+ \text { addition } \text {. }
$$

The calculation is carried out as shown in Figure 1, and the code snippet that follows, here using a cutoff of $30 \mathrm{mV}$ and $1 \mathrm{~ms}$ time step with the previous point at 0 and current point at 1 . For the green line, the brackets show $A=20$ and $B=9 \rightarrow$ spiked in the first third (just) and for the red line $A=2$ and $B=24 \rightarrow$ spiked in the last third:

\footnotetext{
${ }^{1}$ The first (multiply + compare) operation is always called, and the second is called in two-thirds of cases.
} 


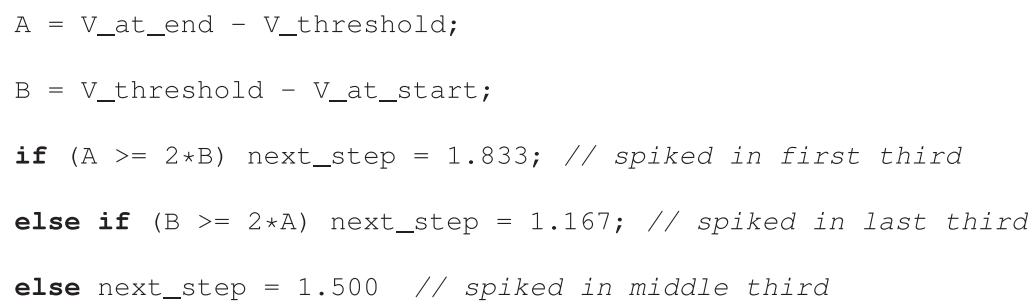

In the comparisons shown later, this scheme is described as TQ3 and the previous simpler scheme as TQ1. Other types of scheme can come about as an automatic result of locally adaptive time steps that are designed to make the solvers more stable just prior to a spike event. These have been investigated briefly with promising results and are mentioned in section 8 .

It should be noted that due to the current clock-driven simulation infrastructure on SpiNNaker, we can currently deliver spikes on the time grid chosen only when the simulation is run. TQ is therefore only an attempt to avoid the lag that would otherwise accumulate in state variable trajectories, without being able to make the spike timings themselves more precise-although this is still going to be beneficial over a long simulation. As Hansel, Mato, Meunier, and Neltner (1998) pointed out, the usual benefits of higher-order methods will be reduced by this limitation unless some method of interpolating the spike timings themselves can be implemented. This is something that deserves further investigation in terms of both which neural models and which solvers are most susceptible; recurrently connected populations are likely to be particularly sensitive. One possible solution under investigation is to use the interpolation method to put a time stamp on a spike when it is sent and therefore recover something like the full potential of using smaller time steps.

\section{Accuracy Results}

Even with the trimmed-down selection of methods described, it would be easy to generate a quantity of data that are difficult to summarize. For example, the solver methods chosen are families rather than individuals, the four parameters in the Izhikevich model allow a very large range of behavior, there are four arithmetic types (32-bit: floating point $\Rightarrow$ float; fixed point $\Rightarrow$ accum, 64-bit: floating point $\Rightarrow$ double; fixed point $\Rightarrow$ long accum) and at least two time steps of interest, and the neuron can be stimulated in different ways, for example. To avoid this explosion of information, a number of decisions have been taken:

- One reference neuron type has been chosen for most evaluation, with limited results for two other examples. It is described by Izhikevich as regular spiking and uses the following parameters: $a=0.02$, $b=0.2, c=-65 \mathrm{mV}, d=8 . V$ and $U$ are initialized at $-75 \mathrm{mV}$ and 0 , respectively. 
- The reference result in each case uses the sophisticated ODE solver in Mathematica, which automatically uses adaptive time steps and handles threshold crossing and stiffness transparently. We have enforced 25 decimal place arithmetic to ensure high accuracy.

- Two time steps are used: $1 \mathrm{~ms}$ for most evaluations and some at $100 \mu \mathrm{s}$.

- The two 32-bit arithmetic types are focused on: accum and float. If the 64-bit types (double and long accum) demonstrate particular behavior, they are referred to specifically.

- Two different stimulations are used. The neuron initially receives no input; then (1) at $60 \mathrm{~ms}$, a $4.775 \mathrm{nA}$ DC current is delivered and sustained (DC input) or (2) at $50 \mathrm{~ms}$, an exponentially decaying input with a time constant of $8 \mathrm{~ms}$ (giving a total charge of $\simeq 80 \mathrm{pC}$ ) is injected and then every $50 \mathrm{~ms}$ thereafter (synaptic input). Once the initial transient settles, these values provide a spike rate of about $10 \mathrm{~Hz}$, exact interspike intervals of $100 \mathrm{~ms}$ that can then be easily compared to the reference. The DC input is a more rigorous test of the accuracy performance of the ODE solvers.

- Results are in the form of graphs that show (1) the membrane voltage $V$ against the reference from 50 to 350 milliseconds and (2) accumulated lags measured in milliseconds for the times of the $n^{\text {th }}$ spike compared to the reference over the first 2 seconds of the simulation (for the more difficult DC input only; all methods perform quite well for the synapse input simulation). In the membrane voltage graphs, the focus should be on the spike timings and the shape of the subthreshold behavior as opposed to the height of $V$ before spiking, as the latter is mainly a function of the time sampling interval chosen for producing the graphs.

A naming scheme for identifying any particular result is the following:

Method-order(type)-ODE or ESR-TQ-Time step in $\mu$ s-arithmetic type

To give an example, RK-3(Heun)-ODE-TQ1-1000-accum means "RungeKutta 3rd order with Heun parameters implemented as an algorithmic ODE solver with the simple time quantization correction at $1 \mathrm{~ms}$ time step using the accum arithmetic type." For ODE solvers, results from the use of static inline functions are not shown separately as they should have an effect only on efficiency, not accuracy.

Solver methods chosen for investigation are the following:

Euler $\equiv$ Runge-Kutta 1st order $\equiv$ Taylor Series 1st order: This is the simplest explicit solver available and the default choice in a number of neural software packages.

Runge-Kutta 2nd order: This is a family of solvers, and so three of the most commonly used members are chosen. The names of this family 
Table 1: Second-Order Runge-Kutta Solver Definitions.

\begin{tabular}{ll}
\hline$a_{2}$ & \multicolumn{1}{c}{ Name } \\
\hline 1 & Trapezoid/improved Euler \\
$2 / 3$ & Ralston/Heun \\
$1 / 2$ & Midpoint/improved polygon/modified Euler \\
\hline
\end{tabular}

are often confused in the literature and so will be referred to as in section 4.3 of Lambert (1973), where they are parameterized by $a_{2}$ as shown in Table 1 and we shall use the names in bold.

Runge-Kutta 3rd order: Another family of solvers and this time with two free parameters. They are generally not confused in the literature and so we refer to them by their common names: Kutta, Heun, Lotkin. As these are inevitably going to be less efficient, we do not investigate them as thoroughly as the second-order methods.

The Runge-Kutta second- and third-order methods can be implemented using ESR or in the standard ODE solver framework. There is much theory related to which member of each family of methods is supposed to be superior, with the choice dependent on how this superiority is assessed. For example, the Ralston second-order method and Lotkin third-order method have their free parameters chosen in order to minimize local truncation error. However, in the presence of finite precision and other implementation details of actual algorithms, it seems that these theoretical results do not necessarily translate into actual benefits.

Taylor Series second- and third-order methods are implemented using ESR and the equations that arise out of the Alsaker (2009) approach.

We have also briefly investigated the methods of Chan and Tsai (2010), which combine Runge-Kutta methods with higher derivatives, with interesting results. The second-order method (which we call CT-2-ESR) produces an identical equation to TS-2-ESR and so is equivalent. The third-order method is new.

6.1 First- and Second-Order methods. Figures 2 and 3 gives a comparison of the spike lags compared to the reference for the first- and selected second-order methods using the DC input and the two 32-bit types (accum use blue lines; float use red lines). Note that each graph uses a different $y$-scale to provide the best precision.

Unsurprisingly, RK1 (=Euler=TS1) is the poorest performer. Of the second-order methods, RK-2 (trapezoid) is a clear winner with accum and equally best with float. Clearly the use of float improves performance over accum here (and in all subsequent results). TS-2 is average but appears to be more susceptible to random numerical errors, as evidenced by the apparent variation around the linear lag trends produced by the RK methods. At 


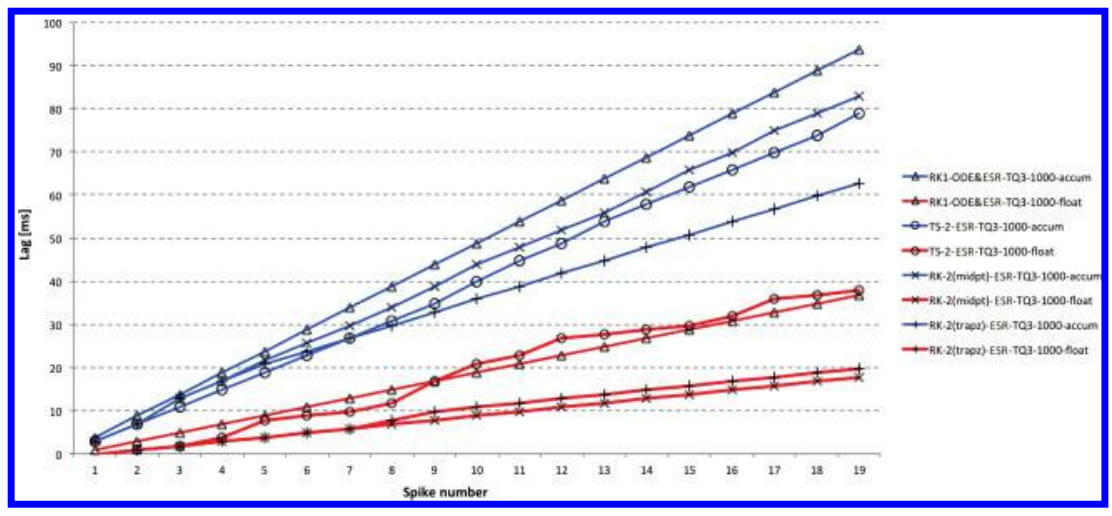

Figure 2: Spike lag for DC input at $1 \mathrm{~ms}$ time step with accum and float types using blue and red, respectively. Float clearly outperforms accum and RK-1 = Euler is the worst-performing solver, although TS-2 performs surprisingly poorly by comparison with float, the apparent random variation almost certainly caused by numerical issues in one of the interim calculations. RK-2 (Trapezoid) is clearly best for accum and approximately equally best for float.

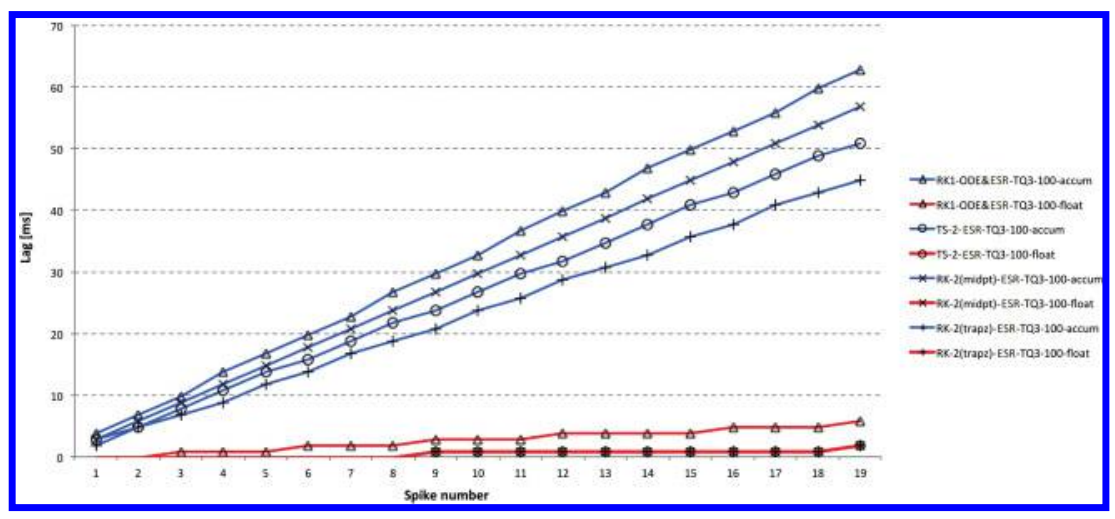

Figure 3: Spike lag for DC input at $100 \mu$ s time step with accum and float types using blue and red, respectively. Float now outperforms accum more clearly and RK-1 = Euler is now consistently the worst performer. RK-2 (trapezoid) is again best for accum and equally best for float. Any of the RK-2 float results at $100 \mu \mathrm{s}$ are likely to be acceptable for high-quality simulations.

$100 \mu \mathrm{s}$, improved lag performance (about $45 \%$ better) with the same approximate ordering is present with accum. With float, all the methods perform very well and identically, apart from RK-1, which is not as good.

Despite being designed to minimize, local truncation error, RK-2 (Ralston) is less accurate than the other two RK-2 algorithms with accum and 
identical with the other three types. As it is also slower, we have not shown these results and focus on the others from here on. The ODE versions of the ESR algorithms shown here are all slightly less accurate for accum and usually identical for the other types.

The next two sets of Figures 4 and 5, and 6 and 7 (one for each input of the stimulation types), show detail from the membrane voltages (i.e., state variable $V$ ) against the reference from 50 to $350 \mathrm{~ms}$. The first in each set compares different solvers at $1 \mathrm{~ms}$ time step and with accum type. The second shows the effect of changing time step and 32-bit type with the RK-2 (trapezoid)-ESR solver.

The lags measured in the previous figures can be seen in the membrane voltages, but over the short timescale used, the separation between the methods is not clear. RK-1 shows an undershoot after the spike event. This is explained by the fact that Euler is in simple terms a linear extrapolation and so will inevitably overshoot a state variable trajectory that contains a substantial second-order component that is heading in the opposite direction-as the initial recovery after a spike will be for the membrane voltage state variable in most integrate-and-fire models.

The comparative benefits from reduced time step and floating point are shown clearly in Figure 5. The benefit of float at $1 \mathrm{~ms}$ is clear (orange versus red), and float at $100 \mu \mathrm{s}$ is very difficult to distinguish from the reference. An interesting comparison is between float at $1 \mathrm{~ms}$ and accum at $100 \mu \mathrm{s}$ (orange versus green). The former is likely to be faster, but the significantly better performance suggested by the lag plots is apparent.

The synaptic input is an easier test, and the lag performance is seen as very similar between the methods. RK-1 still shows overshoot after a spike event in Figure 6. The various methods follow the pulse at 150 and $250 \mathrm{~ms}$ with varying degrees of fidelity.

With RK-2 (trapezoid) the benefits of shorter time step and floating point are less clear with the synaptic input in Figure 7.

6.2 Third-Order Methods. For comparison, a selection of third-order methods is also shown despite the extra computational load that will inevitably result. (See Figures 8 and 9.)

It was quite a surprise to see how badly the standard third-order methods performed at $1 \mathrm{~ms}$ time step, even with the 64-bit types. Of the Runge-Kutta third-order order methods, only RK-3 (Heun) provides reliable solutions on the DC input, and even then they are less accurate than the second-order methods. With accum, only the ESR methods worked at all. Previous experience with ODE solvers has shown an incremental improvement with increased order up to fifth and sixth order, as predicted by analytical results in standard texts. However, this experience has usually been with adaptive time-step algorithms and double-precision floating point.

Some diagnostics were therefore required to investigate an initial hypothesis of underflow or overflow (or both), and for this, a range-checking 


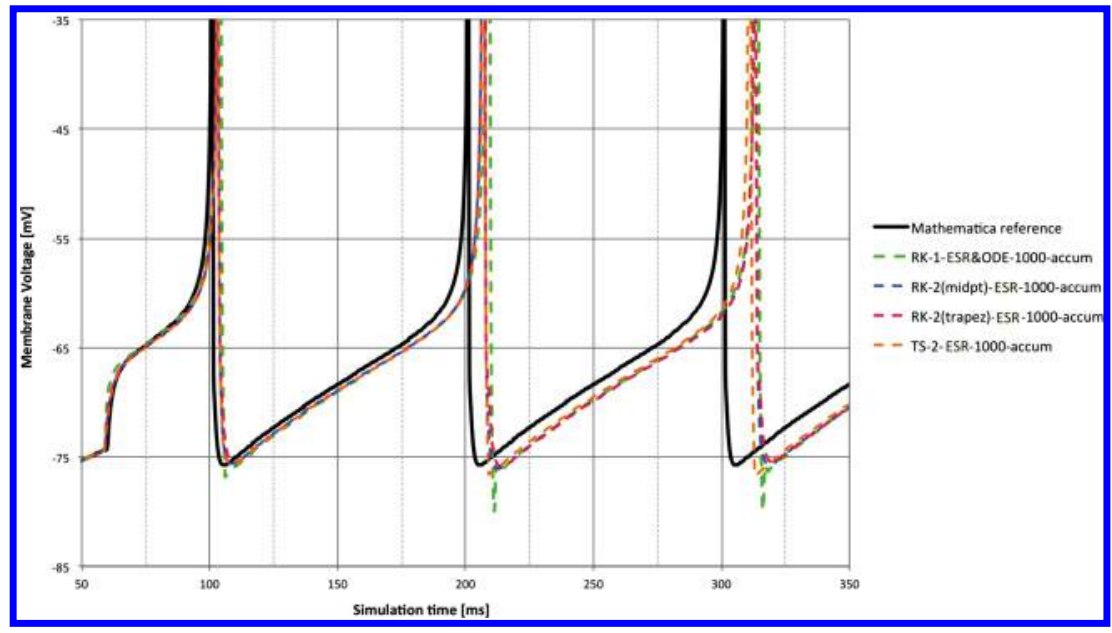

Figure 4: Membrane voltage for DC input at $1 \mathrm{~ms}$ time step and accum type compared to reference. The spike lags measured in Figure 2 are clear. Note the overshoot after the spike with the RK-1 solver.

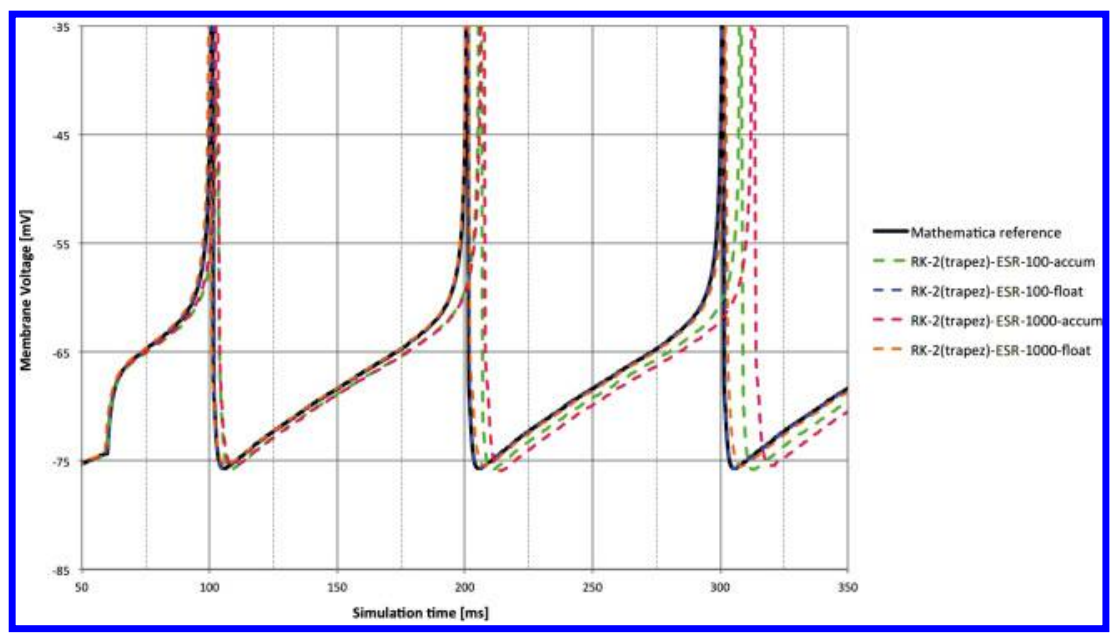

Figure 5: Membrane voltage for DC input. RK-2 (Trapezoid) ESR solver with different 32-bit arithmetic types and time steps compared to reference. The $100 \mu \mathrm{s}$ and float combination is difficult to distinguish from the reference, and even at $1 \mathrm{~ms}$, float provides good performance-significantly better than accum at $100 \mu \mathrm{s}$. 


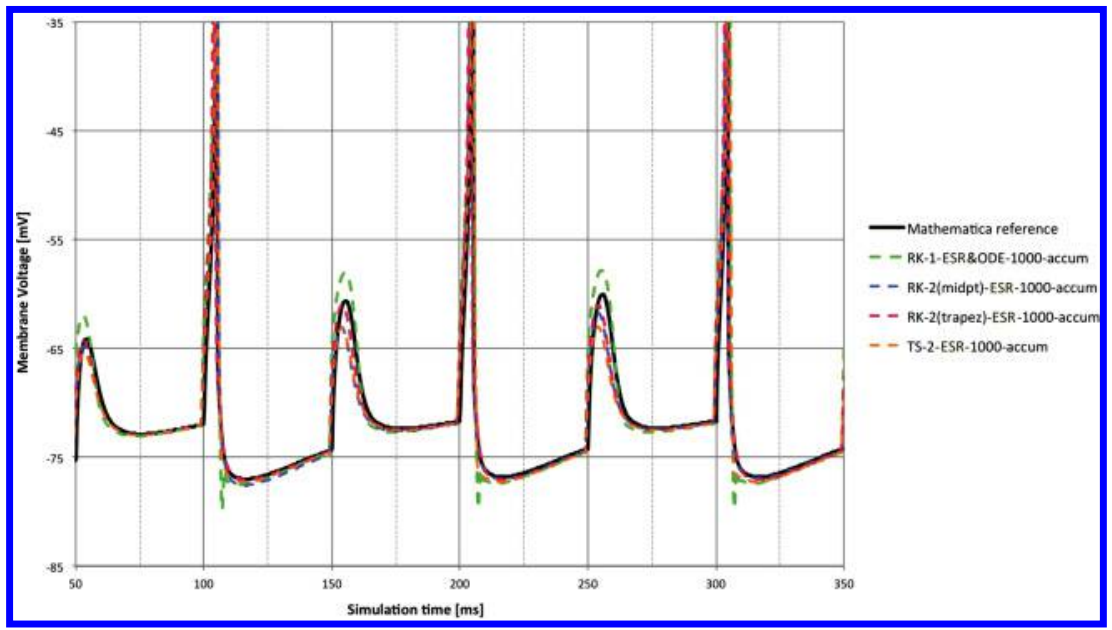

Figure 6: Membrane voltage for synaptic input at $1 \mathrm{~ms}$ time step and accum type compared to reference. Clearly this is an easier task than DC input, with all methods giving relatively good performance except some overshoot behavior from RK-1 = Euler.

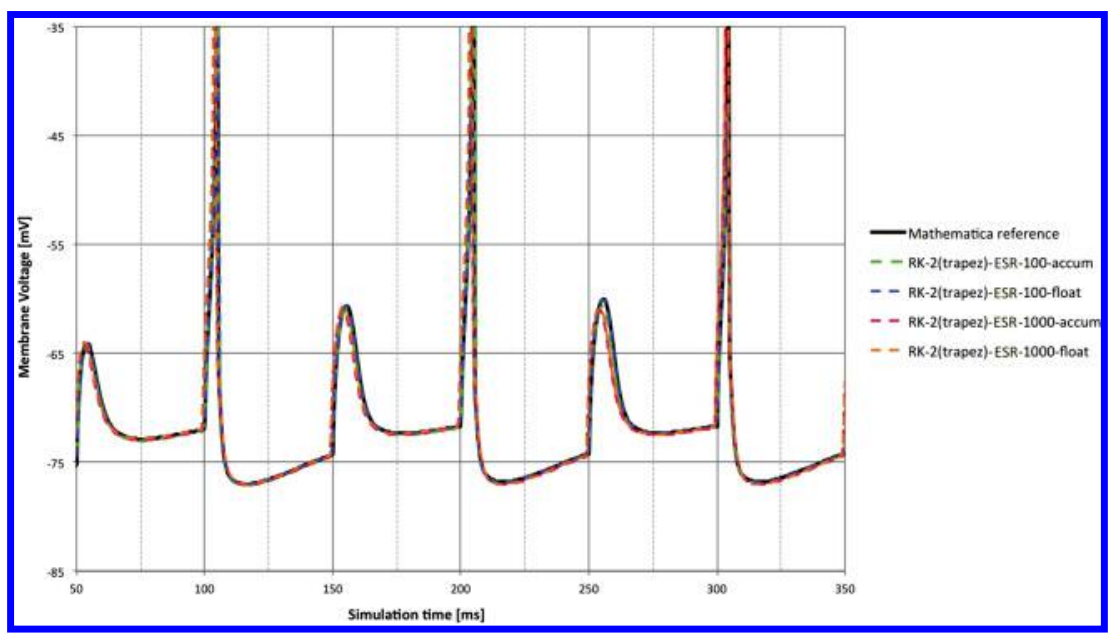

Figure 7: Membrane voltage for synaptic input. RK-2 (Trapezoid) ESR solver with different 32-bit types and time steps compared to reference. A good performance in all cases, with the $100 \mu$ s results difficult to distinguish from the reference. 


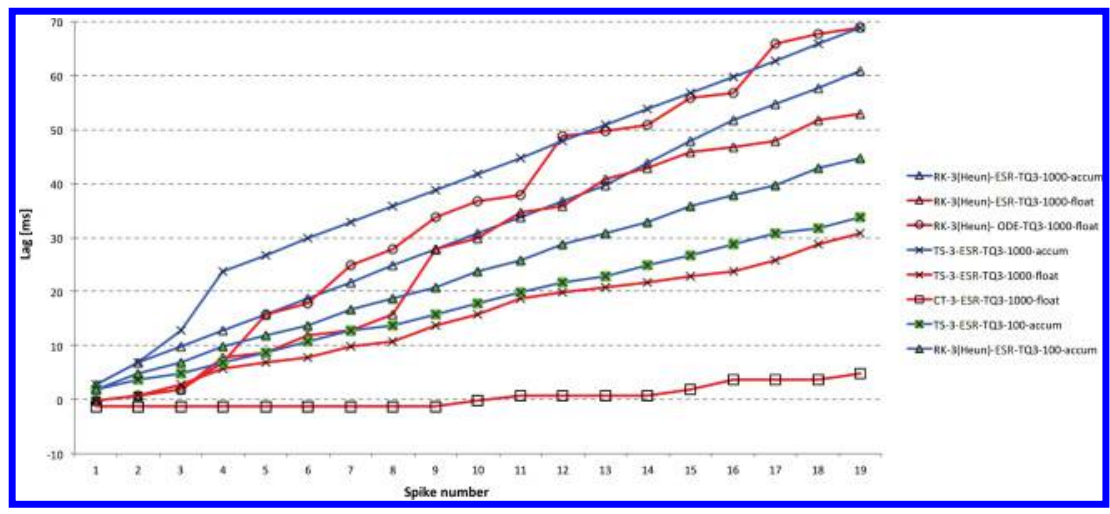

Figure 8: Spike lag for DC input for various third-order combinations of solver and time step, with accum and float types using blue and red, respectively. A surprisingly poor set of results, especially for float in some cases, caused by numerical issues discussed in the text. CT-3 gives excellent performance at $1 \mathrm{~ms}$ in this case with little efficiency penalty compared to RK-2 solvers.

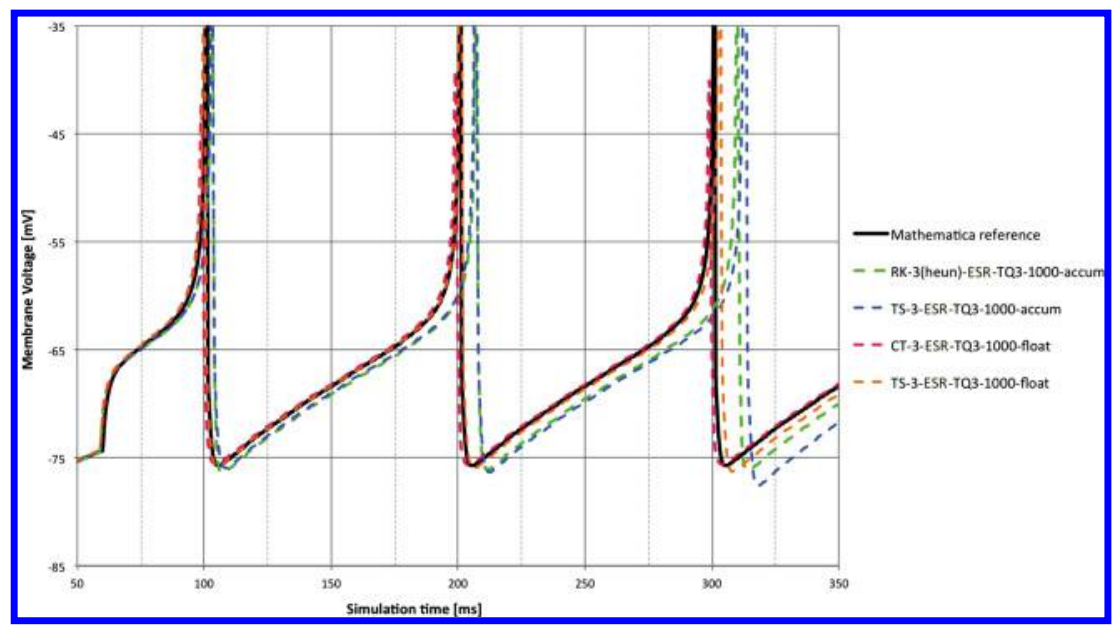

Figure 9: Membrane voltage for DC input at $1 \mathrm{~ms}$ time step with various thirdorder combinations of solver and arithmetic type. Poorer-than-expected performance is again shown here. CT-3 (and to a lesser extent TS-3) with float are a good match to the reference.

macro was formulated that could print out values of selected state variables or intermediate values internal to the algorithms if they got either very small or very large. This macro works for all arithmetic types and can be removed in one place for speed testing. It shows that with the higherorder RK methods, intrastep predictions and derivatives calculated during 
(and particularly toward the end of) a time step can become enormous just before a spike event. One reason that the higher-order RK methods suffer more from this phenomenon is that they make predictions or calculate derivatives later in the time step than lower-order methods, where these effects become rapidly more prevalent near a spike. Plotting high-order derivatives of the reference solution show very large values in these cases, which will either overflow the arithmetic type or violate assumptions made when assessing local error behavior in the presence of exact arithmetic. In essence, the membrane voltage exhibits superexponential behavior near cutoff that can not be modeled well when a relatively large fixed time step is used in the solution, and the higher-order methods suffer more as they attempt to track these very large high-order derivatives. With $100 \mu \mathrm{s}$ time step, no problems were seen with any of the algorithms.

Methods that locally switch to smaller time steps when close to spikes have been investigated briefly and show promising results. They clearly improve the performance of the third-order methods described here with a small speed penalty and give an opportunity to get a better estimate of threshold-crossing time than TQ3 if formulated correctly. It may be worthwhile to pursue these ideas further, as they will be of some benefit to all solvers if the speed penalty can be made small enough.

Although the Runge-Kutta methods are widely considered to be the method of choice where singularities are likely to be encountered in the solutions (see, e.g., chapter 16 of Press et al., 1992), some have mentioned the dangers of applying the higher-order versions in such cases (p. 232 of Gear, 1971, and p. 21 of Enright, Higham, Owren, \& Sharp, 1995). We conclude that this, in combination with the restriction of a fixed time step that is too large to allow effective tracking of the superexponential behavior near to a spike and, to a lesser extent, finite precision issues, are the causes for the higher-order methods being so ineffective here.

6.3 Other Izhikevich Neuron Types. In Figures 10 to 12, we show some comparison of membrane voltages against reference result for the RK-1 and RK-2 (Trapezoid) solvers (the latter with different time steps and arithmetic types) on two other types of Izhikevich neuron using the DC input.

The types chosen are:

1. Chattering $\Rightarrow\{a=0.02, b=0.2, c=-50, d=2\}$

2. Fast Spiking $\Rightarrow\{a=0.1, b=0.2, c=-65, d=2\}$

Both of these neuron types are going to be harder for the ODE solvers to deal with because of higher rates of change relative to the fixed time step. For the Chattering neuron, these high rates of change come in clusters; for the Fast Spiking neuron, the rate of spiking is just uniformly higher for a given input.

With the Chattering neuron, it is clearly far more difficult to assess the detail of the spiking behavior when clusters of spikes are being generated. 


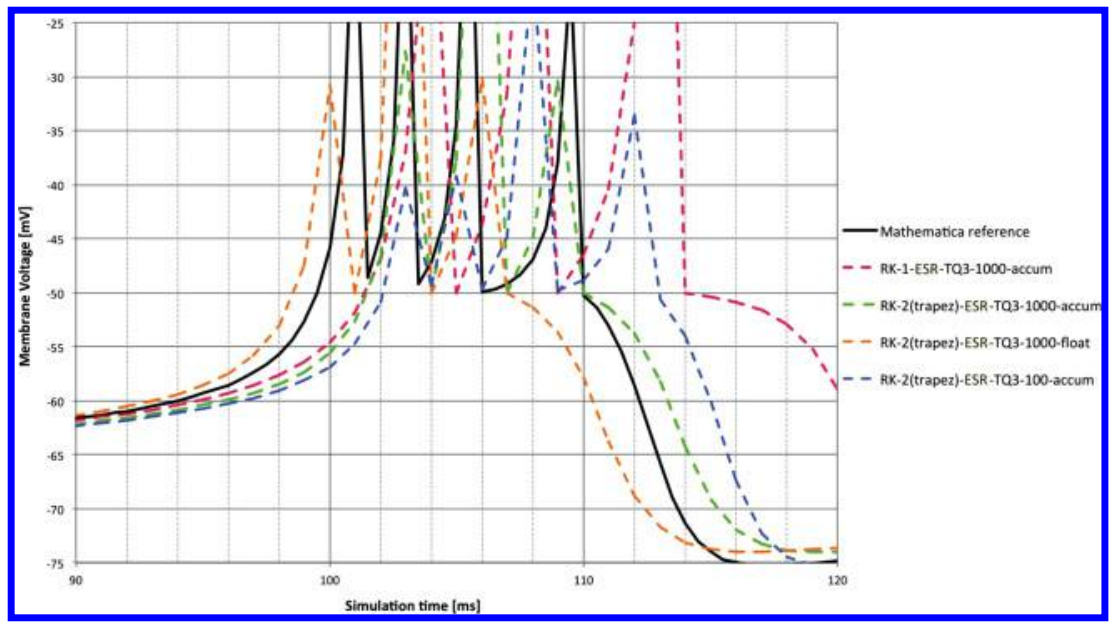

Figure 10: Membrane voltage for DC input with varying solver setups and arithmetic types compared to reference. Chattering neuron (zoomed time axis to show more detail). Both leading and lagging behavior present with only the $100 \mu$ s result providing four spikes per burst as reference. RK-1 = Euler is the worst performer

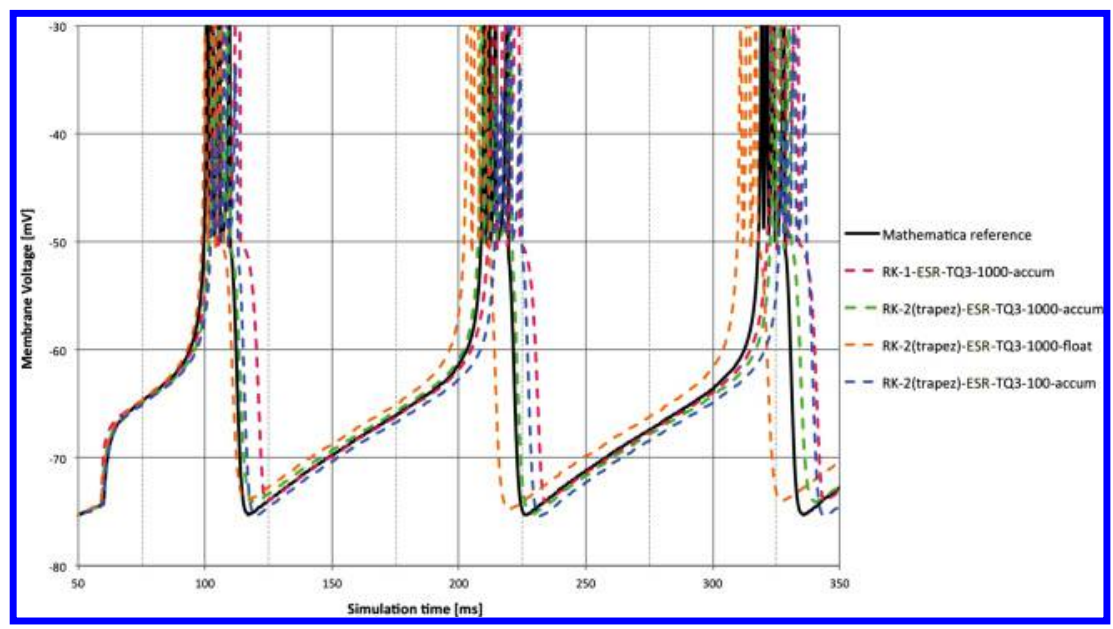

Figure 11: Membrane voltage for DC input with varying solver setups and arithmetic types compared to reference. Chattering neuron over normal timescale. This is clearly a challenging task and no clear winner. The $100 \mu \mathrm{s}$ result produces the correct number of spikes per burst but starts to lag reference. 


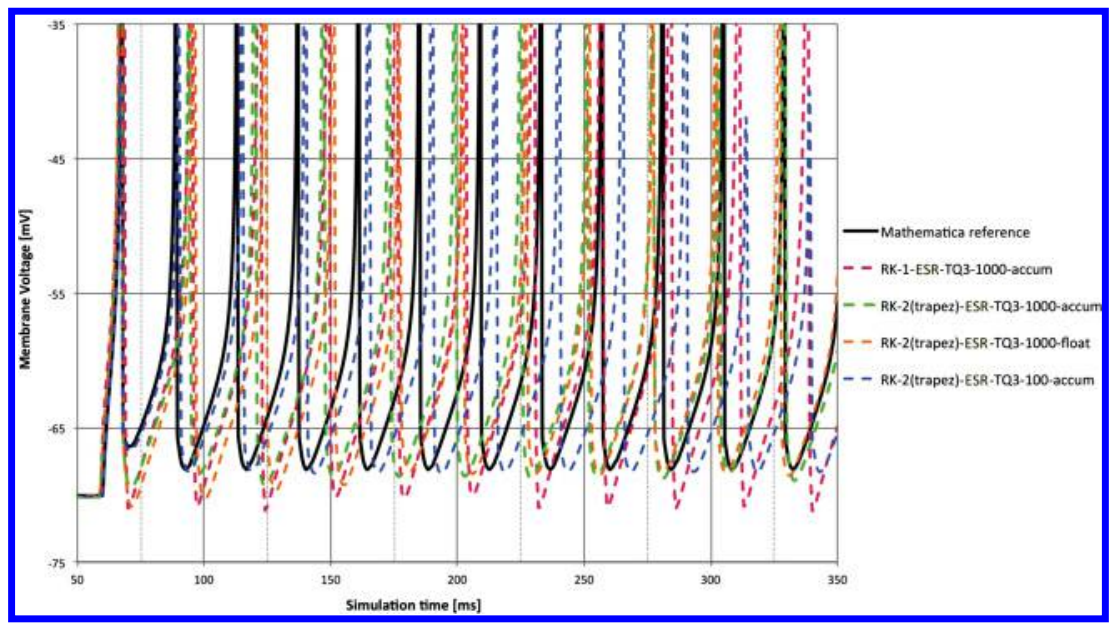

Figure 12: Membrane voltage for DC input with varying solver setups and arithmetic types compared to reference. Fast Spiking neuron. The $1 \mathrm{~ms}$ results all lag more than the $100 \mu \mathrm{s}$ accum result. RK-1 = Euler gives overshoot and the worst lag.

Figure 10 zooms in on the time between $90 \mathrm{~ms}$ and $120 \mathrm{~ms}$ to improve clarity for the first cluster. Only RK-2 (Trapezoid) at $100 \mu$ s time step manages to match the four spikes produced by the reference solver, albeit with some lag. The $1 \mathrm{~ms}$ solvers all produce three spikes, with RK-2 (Trapezoid)-float clearly leading and RK-1-accum clearly lagging. Figure 11 on the usual time axis shows some patterns that are present in the lag behavior that differ from those seen on the reference neuron. Although the worst lag is RK-1-accum as before, RK-2 (Trapezoid) at $100 \mu$ s time step is not much better and appears to be getting worse as time goes on, which is a surprise as it works well with the reference neuron. The tendency for RK-2 (Trapezoid)-float to lead the others is also shown here, but it seems to be leading too much, at least over the first $350 \mathrm{~ms}$. Perhaps surprisingly, the best performer in terms of overall lag seems to be the RK-2 (Trapezoid)-accum at $1 \mathrm{~ms}$ time step.

For the Fast Spiking neuron in Figure 12, the story is a little simpler. All the 1 ms time step results lag the reference results with RK-1 worst, then RK-2 (Trapezoid)-float and RK-2 (Trapezoid)-accum quite similar. RK-2 (Trapezoid)-accum at $100 \mu$ s stands apart as lagging far less-about $10 \mathrm{~ms}$ by the twelfth spike. Clearly the smaller time step deals better with the higher spike rate, which is not surprising.

6.4 Discussion of Accuracy Results. In all of the cases tested, the 64-bit types produce results similar or identical to the 32-bit float type, and so no separate figures have been produced. This suggests that no significant 
benefits would accrue in these cases, and therefore the computation and storage cost of 64-bit types are not currently justified.

It is worth noting that for synaptic input (which looks on the face of it to be the more realistic case), the use of accum and $1 \mathrm{~ms}$ does not have much of a penalty associated with it even for the simpler methods. However, although neurons in a realistic network could be receiving inputs from (in some cases in the auditory system) a single synapse, more commonly it would be up to 1000 synapses or more, and for the latter case, the summation of these inputs at realistic spike rates and $1 \mathrm{~ms}$ time step would converge asymptotically on DC input with gaussian noise. This makes the case for the more challenging input type in terms of simulating actual behavior. Results in Mainen and Sejnowski (1995) and Brette and Guigon (2003) suggest that some aperiodic variation around a DC component could be a good deterministic test signal in future work. As we mention later, simulating realistic amounts of pseudorandom input noise and analyzing output distributions in the presence of this noisy input would be closer still to a realistic use case.

Clearly, to find the best ODE solver, one needs to work with a realistic set of neural behaviors. From the brief analysis of two other common types in the previous section, it seems that a solver that works well on one neuron type does not necessarily transfer that superior performance to other types. Although useful as another demonstration of performance characteristics, it should be remembered that the other two neuron types are a harder challenge and that these spike rates are less likely in spiking neural networks that match realistic behavior in much of the brain (though they can exist transiently or in certain areas). In any case, the complex interaction between neuron type, time step, arithmetic type, solver algorithm, and even exact ordering of arithmetic operations make a definitive statement difficult to reach here. Some patterns are, however, present. The second-order methods generally improve on RK-1, in some cases significantly. A more complete investigation would require the capability to script and automate test cases in order to search the various parameter spaces more thoroughly.

The almost linear nature of some of the spike lag plots might lead one to believe that a simple correction could be applied in order to bring this line back onto the $x$-axis. However, the observed lag is a complex and nonlinear function of input dynamics and neuron definition among other things, and solving this problem would be computationally more challenging than simply moving to a better solver.

\section{Efficiency Results}

The results in Table 2 show the time taken for a SpiNNaker board running at $200 \mathrm{MHz}$ to carry out 10,000 neuron updates in a simple loop, divided by 10,000 . The synaptic input scheme is used with $1 \mathrm{~ms}$ time step. After the initial transient, the spiking rate $\simeq 10 \mathrm{~Hz}$ so that discrete state updates are in correct relative proportion. The timing is corrected to remove the 
Table 2: Cost in $\mu$ s per Neuron Update with TQ1 Correction.

\begin{tabular}{|c|c|c|c|c|}
\hline & accum & float & long accum & double \\
\hline RK-1 (三Euler; ODE $\equiv \mathrm{ESR})$ & 0.56 & 3.99 & 6.48 & 6.35 \\
\hline RK-2 (MidPoint)-ESR & 0.86 & 6.97 & 12.54 & 11.23 \\
\hline RK-2 (Trapezoid)-ESR & 0.97 & 7.67 & 13.41 & 12.30 \\
\hline RK-2 (Ralston)-ESR & 1.07 & 8.29 & 15.87 & 13.52 \\
\hline TS-2-ESR $\equiv C T-2-E S R$ & 0.93 & 6.81 & 13.22 & 10.90 \\
\hline RK-2 (MidPoint)-ODE gen driver & 2.33 & 8.91 & 14.41 & 13.64 \\
\hline RK-2 (MidPoint)-ODE gen driver S.I. & 1.36 & 7.68 & CompErr & 11.95 \\
\hline RK-2 (MidPoint)-ODE spec driver & 1.63 & 8.21 & 13.67 & 13.01 \\
\hline RK-2 (MidPoint)-ODE spec driver S.I. & 0.89 & 7.24 & 12.61 & 11.66 \\
\hline RK-2 (Trapezoid)-ODE gen driver & 2.37 & 9.46 & 14.42 & 14.50 \\
\hline RK-2 (Trapezoid)-ODE gen driver S.I. & 1.32 & 8.21 & CompErr & 12.87 \\
\hline TS-3-ESR & 2.19 & 13.46 & 28.30 & 21.84 \\
\hline RK-3 (Heun)-ESR & 1.50 & 11.43 & 21.96 & 18.78 \\
\hline RK-3 (Heun)-ODE spec driver & 2.16 & 10.47 & 21.85 & 21.33 \\
\hline RK-3 (Heun)-ODE spec driver S.I. & 1.26 & 11.57 & 20.35 & 19.06 \\
\hline CT-3-ESR & 1.86 & 11.12 & 28.40 & 15.80 \\
\hline
\end{tabular}

Notes: The static inline functions are denoted S.I.; general ODE drivers with function pointers are denoted gen driver; the most specialized drivers are denoted spec driver. CompErr means that a compiler error caused a build failure.

overhead of code required for generating input, collecting and counting spikes, and other administrative functions. The time per update therefore refers only to the cost of computation and memory access and movement specific to each algorithm. The compiler flag -Ofast is used, and thumb code is disabled to promote speed above other considerations and allow the compiler to perform as many optimizations as possible. ${ }^{2}$ No manual adjustment of assembly code is used, though preliminary investigations have shown potential for this to improve performance, for example, in the case where the multiplication of different fixed-point types might deliver improved accuracy. Floating-point operations on the ARM968 processor are carried out by software library functions.

For the ODE solver results, we have a number of comparisons available for the same methods: (1) implemented either as either normal C source or static inline functions in the header files (these are denoted S.I.) and (2) the difference between the most general ODE driver that uses function pointers (gen driver) and the most specialized one that explicitly codes the algorithm and avoids inner loops by knowing that two state variables are present (spec driver). CompErr means that a compiler error does not allow that build to complete.

\footnotetext{
${ }^{2}$ The compiler used is the gcc 4.7 .3 cross compiler from Mac OS X to arm-none-eabi.
} 
7.1 Discussion of Efficiency Results. With RK-1 as the reference and for the moment considering only accum results, the extra cost of a secondorder method is between $54 \%$ and $91 \%$ for ESR, between $59 \%$ and $143 \%$ for ODE in static inline form, and between 191\% and 323\% for ODE using function pointers and general loops over state variables. Clearly the ESR is effective here as well as in terms of accuracy. The static inline results show that the compiler makes effective use of the optimizations allowed across source files that would not otherwise be possible. The fastest second-order method is RK-2 (Midpoint)-ESR. The two fastest third-order methods cost $125 \%$ and $168 \%$.

For float, the relative costs differ somewhat. On average, the solution takes 6.6 times longer, though the relative penalty is uniformly less for the general ODE results, presumably because they were worse in the first place. The faster second-order methods are generally slightly slower relative to RK-1, and the ordering among them is approximately the same except that now, TS-2-ESR is now the smallest penalty at 71\%, though RK-2 (Midpoint)ESR is not far behind at $75 \%$.

Adding TQ3 correction costs typically about 0.025 to $0.05 \mu \mathrm{s}$. This is perhaps larger than expected because the correction is calculated only when a spike occurs-approximately once for every 100 updates, which suggests that the cost of each TQ3 correction is about $5 \mu \mathrm{s}$. Removing TQ correction altogether provides a useful efficiency gain for two reasons: (1) as the fixed time step is now always known in advance, this simplifies the optimal ESR form, leading to less computation, and (2) one indirect memory access is no longer required. For example, the cost of one neuron update for RK-2 (MidPoint)-ESR reduces to $0.73 \mu \mathrm{s}$ for accum, a 15\% improvement. As the algorithm gets simpler, another threshold is reached where such gains become even greater, presumably because the compiler finds it increasingly easy to optimize the use of registers. An example is a stripped-down version of RK-1 with accum where all code related to TQ is removed. The time per update becomes $0.45 \mu \mathrm{s}$, a 20\% gain over the standard TQ1 version.

\section{Conclusion and Ideas for Further Work}

Perhaps with an innovative technology like SpiNNaker, which offers an unexpected combination of very low energy use and real-time performance, it should not be a surprise if some trade-offs need to be made. A comparison with neural solver frameworks that work with double-precision floating-point arithmetic, adaptive time steps, high-order/implicit solvers, and sophisticated threshold-crossing algorithms will inevitably show some differences in terms of state variable trajectories and spike timings. However, with a careful choice of solver and TQ approach, even just the use of float instead of accum with either fewer neurons per core (or, equivalently, performance that is slower than real time) will provide a performance that is very close to these much more energy-intensive systems, which themselves 
offer only some tiny fraction of real-time performance. Other possibilities such as order adaptivity and within-step time adaptivity are discussed below. As with almost everything in the area of mathematical computation, an informed choice of where to be in the accuracy, speed space needs to be made, and we hope that this study helps to elucidate some of the trade-offs.

The Izhikevich neuron model creates membrane voltage trajectories that are super exponential near to the spike with large high-order derivatives, and the spike events are discontinuities. These will always provide a challenge to explicit fixed time-step algorithms with time steps that are significant relative to the rate of change, as they try to track the sudden increase and inevitably lag. This is the main cause of the lagging shown in the accuracy result, and the failure of the higher-order methods to improve on the lower-order ones at $1 \mathrm{~ms}$ time step.

Although full time-step adaptivity has been ruled out for this study, some other options are possible. Parker-Sochacki offers a form of order adaptivity, adding higher-order terms as necessary, and this has been investigated briefly here in the context of adding higher-order terms in the Taylor series solvers-in our case, up to fourth order. It is easy to achieve in this case, as each higher-order update to the state variable can be simply added to the previous one. Current results are promising but variable and so have not been reported in detail yet. It is likely that the decision on when to add higher-order terms is the key to success for this approach, and this requires further study. It is also likely that the higher-order terms will be more difficult to calculate in the presence of numerical errors. A similar approach could be used for Runge-Kutta solvers, but it would inevitably be more expensive computationally or at least much more complex.

Another possibility that shows promise is limited time-step adaptivity, where at certain points, the solver divides its own internal time step by an integer $d$ and then repeats the solve $d$ times before returning the updated neuron to the system. This will obviously create a " $d$ times" overhead, but only at points where it is needed-near spikes where the derivatives get very large-and so is unlikely to generate a significant overhead for the simulation as a whole. As with order adaptivity, the key will be making an effective and robust decision about when to carry it out. Initial experiments show useful improvements for all solvers at relatively modest cost, for example, making the third-order methods more stable.

A simple mechanism for removing the accumulated lag caused by ignoring the actual time of a threshold crossing has been proposed and implemented. The simplest, TQ1, has little overhead and removes the $1 / 2$ time step bias. A more complex one, TQ3, adds only a small extra overhead and provides significantly more accurate post spike transitions with a mean absolute deviation of $1 / 12$ time step. Allowing the size of the time step to be adaptive near spikes for better accuracy and stability, as discussed above, provides a different mechanism for measuring the threshold-crossing time at essentially no extra cost. At the moment, however, neither of these 
mechanisms will deliver spikes between time steps. As the SpiNNaker system evolves, it may be that certain ground rules change; for example, event-driven rather than clock-driven behavior might become possible (see, e.g. section 2.3 of Brette et al., 2007).

Both accuracy and efficiency results are sensitive to exact implementations of the algorithms. Obviously the compiler reorders instructions according to the optimization scheme. In this work, it has been commonly found that the simple reordering of one subtraction in an algorithm that has eighty mathematical operations in total can make a significant difference, especially to accuracy and for the 32-bit types. Also, the neatest algebraic reductions are not always the ones that produce the best accuracy (with $a c$ cum in particular), but they do usually produce the best efficiency. Clearly, the optimization of a particular solution requires art and experimentation as well as science. (See Martel, 2009; Darulova et al., 2013; and Gao et al., 2013, for ideas about how the process could be made more scientific.)

Other neuron models will provide different challenges and opportunities. Complex behavior (e.g., the Hodgkin-Huxley model) will almost certainly require higher-order methods and possibly implicit solvers, with consequences for neuron update performance and the number of neurons that can be supported per core in real time. An effective application of Parker-Sochacki for this model is shown in Stewart and Bair (2009) and the approach may be of wider interest.

Of course, the ideal is to have closed-form solutions (or at least close approximations) for the state variable trajectories as available with, for example, the current-based leaky integrate-and-fire (LIF) neuron. As our ODE is autonomous, the integral is separable, and so a standard solution technique is available:

$$
\frac{d V}{d t}=f(V) \Rightarrow \int d t=\int \frac{d V}{f(V)}
$$

The general requirement for knowing the membrane voltage at the end of the next time step is then the solution of integral equations of the following form, with the unknown in this case being Vnext:

$$
\text { timestep }=\int_{\text {Vnow }}^{\text {Vnext }} \frac{1}{f(V)} d V \text {. }
$$

Some progress has been made on such solutions for one state variable in the Izhikevich equations if the other state variable is kept fixed over the time step. As Humphries and Gurney (2007) showed, this assumption, leads to an exact solution for the $U$ state variable. Using a further assumption, they also show a solution for the $V$ state variable with a slightly more complex version of the Izhikevich equations than ours. We have found an analytical 
solution for our simpler version, which may not have yet appeared in the literature and is given below, where $t$ is in milliseconds and $I$ is in nanoamps:

$$
\text { Vnext }=-5 \sqrt{\alpha} \tan \left(2 \sqrt{\alpha}\left(-\frac{0.5 \tan ^{-1}\left(\frac{\gamma}{\sqrt{\alpha}}\right)}{\sqrt{\alpha}}-0.1 t\right)\right)-62.5
$$

and

$$
\alpha=I-U-16.25, \quad \gamma=0.2 V+12.5 \text {. }
$$

Clearly, $\alpha$ will be negative in many cases, leading to the need for complex arithmetic and using the real part of the solution (which appears to always have zero imaginary part anyway). This does suggest that an even simpler form may be available, but we have not yet found it.

Of course, the extra computation required for the above may not be justified if holding one state variable fixed over the time step in order to solve the other introduces more error than the exact solution removes. Finding a solution for the coupled 2D system of integral equations for both $V$ and $U$ is the real answer, but it has so far proved elusive and may not be possible. It is almost certain that if such a solution exists, it will require the use of transcendental functions and complex numbers, which reinforces the need for a fast and accurate library of such operations for fixed-point types to be available.

It is likely that the ESR will be useful anywhere that the ODE is autonomous and an explicit solver can be used. We have shown that carefully implemented algorithms can be both more accurate and faster than the most heavily optimized general solver of the same kind with static inline function optimization. The fact that the neuron solver code can be delivered pretested and in standard compiled library + header form also provides software engineering advantages.

Due to time and space limitations, we have looked at only a small selection of Izhikevich parameters (and therefore neuron behaviors) in this study, and so it cannot be considered an exhaustive analysis. We hope that the examples we chose show trends that can be extrapolated to more general conclusions, but of course it is possible that unseen pathological cases exist. Even a small sample of two other neuron types within the compass of the Izhikevich parameterization has shown some different patterns from the reference neuron results. One way to achieve better coverage is to automate the testing mechanism. This was partially implemented for the study within the main $C$ event loop running on the SpiNNaker board, but to achieve it comprehensively, the ability to script testing mechanisms on a host would be ideal. At the moment, this is not straightforward using the fixed-point types because they only cross-compile to an ARM target with no operating 
system. If these types could be supported on the host machine-or, alternatively, within a scriptable emulator environment-then validation over larger example spaces in particular would become much easier. It would also facilitate testing with stochastic inputs, which are very likely in actual use cases, leading to output measures based upon differences between distributions of spikes or spike intervals using, for example, Kullback-Leibler information-theoretic measures, with the reference neuron providing the reference output distribution.

Hybrids between multistep, multistage, and multiderivative methods exist and can be reasoned about using the approach of general linear methods as shown in chapter 5 of Butcher (2003), who mentions some of the better-known examples: the Rosenbrock and Obreshkov methods. The extent to which these hybrid methods can find a better balance between accuracy and efficiency while retaining stability and remaining within our nominal constraints of fixed $1 \mathrm{~ms}$ time step and accum arithmetic type has not yet been fully explored, but the potential is there-particularly those combining multistage and multiderivative approaches (usually called Obreshkov or Turan methods). The methods discussed in Chan and Tsai (2010) should be worthwhile investigating further as they allow an algebraic combination of our Taylor series and Runge-Kutta solutions for potentially better accuracy at minimal extra overhead. Initial tests have been very promising, with CT-3-ESR being by far the most accurate algorithm at $1 \mathrm{~ms}$ time step (except for the accum type, where numerical underflow sabotages accuracy in all the algorithm orderings so far investigated) while also being the fastest third-order algorithm for floating-point types. (For related work see Shintani, 1972; Mitsui, 1982; and Ono \& Yoshida, 2004.)

At the current time and on the basis of the limited set of tests so far carried out on Izhikevich neuron types, the best trade-off between speed and accuracy-using the accum type and $1 \mathrm{~ms}$ time step to facilitate realtime operation-looks to be one of the RK2-ESR solver methods; Midpoint for speed or Trapezoid for accuracy.

\section{Appendix: An Example of ESR}

This appendix shows an example of the ESR, from an original definition of the ODE and explicit solver algorithm, through a reduction to constituent common terms, and ending up in $\mathrm{C}$ source code. The analysis and reduction is carried out using Mathematica. We start with a definition of the Izhikevich equation as derivatives with regard to $V$ and $U$ as follows:

$$
\begin{aligned}
& \operatorname{ODEV}(V, U)=I-U+((0.04 V+5) V+140), \\
& \operatorname{ODEU}(V, U)=a(b V-U) .
\end{aligned}
$$

Next we define an explicit ODE solver formula. In this case, it will be the Runge-Kutta second-order midpoint method (sometimes also called 
modified Euler):

$$
\begin{aligned}
& V \rightarrow V+h \operatorname{ODEV}\left(V+\frac{h}{2} \operatorname{ODEV}(V, U), U+\frac{h}{2} \operatorname{ODEU}(V, U)\right), \\
& U \rightarrow U+h \operatorname{ODEU}\left(V+\frac{h}{2} \operatorname{ODEV}(V, U), U+\frac{h}{2} \operatorname{ODEU}(V, U)\right) .
\end{aligned}
$$

Combining these generates algebraic formulase equivalent to running the RK-2 (Midpoint) ODE solver with the Izhikevich ODE system. So for the next value of $V$ and $U$, we have:

$$
\begin{aligned}
V \rightarrow & V+h\left(140+I-U-\frac{h}{2} a(-U+b V)\right. \\
& +\left(V+\frac{h}{2}(140+I-U+(5+0.04 V) V)\right) \\
& \left.\left(5+0.04\left(V+\frac{h}{2}(140+I-U+(5+0.04 V) V)\right)\right)\right), \\
U \rightarrow & U+a h\left(-U-\frac{h}{2} a(-U+b V)\right. \\
& \left.+b\left(V+\frac{h}{2}(140+I-U+(5+0.04 V) V)\right)\right) .
\end{aligned}
$$

Even for a relatively simple second-order ODE solver, these equations are quite unwieldy. As the order increases, they rapidly become unmanageable in this form. Fortunately, there are many ways of simplifying them and finding common terms, typically either automatically using the algebraic manipulation commands available in Mathematica, by inspection, or usually a combination of the two. Considerations are:

- Maximizing the number of common terms among the state variables to minimize computational load

- For fixed-point types, ensuring that interim variables have values that minimize the possibility of under- or overflow, around 2.0 for accum

- In general, choosing interim calculations that minimize truncation or roundoff errors

- Orderings of arithmetic operations can have an effect on both accuracy and speed (though this is due to the C compiler later in the chain)

One choice of common terms is described by the set of Mathematica substitutions given below. These were arrived at after experimenting with a range of different possibilities. It provides the RK-2 (Midpoint)-ESR solver that is both the fastest and most accurate among a number tested so far across a range of arithmetic types and therefore seems to be close to optimal 
within the limited testing regime:

$$
\begin{aligned}
<\text { combined equation }>/ / . & \{140+I-U \rightarrow \theta, \theta+(5+0.04 V) V \rightarrow \alpha, \\
& \left.\frac{h}{2} \alpha+V \rightarrow \eta,-\frac{h}{2} a(-U+b V) \rightarrow \beta\right\} .
\end{aligned}
$$

Applying this substitution to the two equations for the state variable updates produces the following expressions:

$$
\begin{aligned}
& V \rightarrow V+h(\beta+(0.04 \eta+5) \eta+\theta), \\
& U \rightarrow U+a h(b \eta+\beta-U) .
\end{aligned}
$$

Translating these into $\mathrm{C}$ code gives the following:

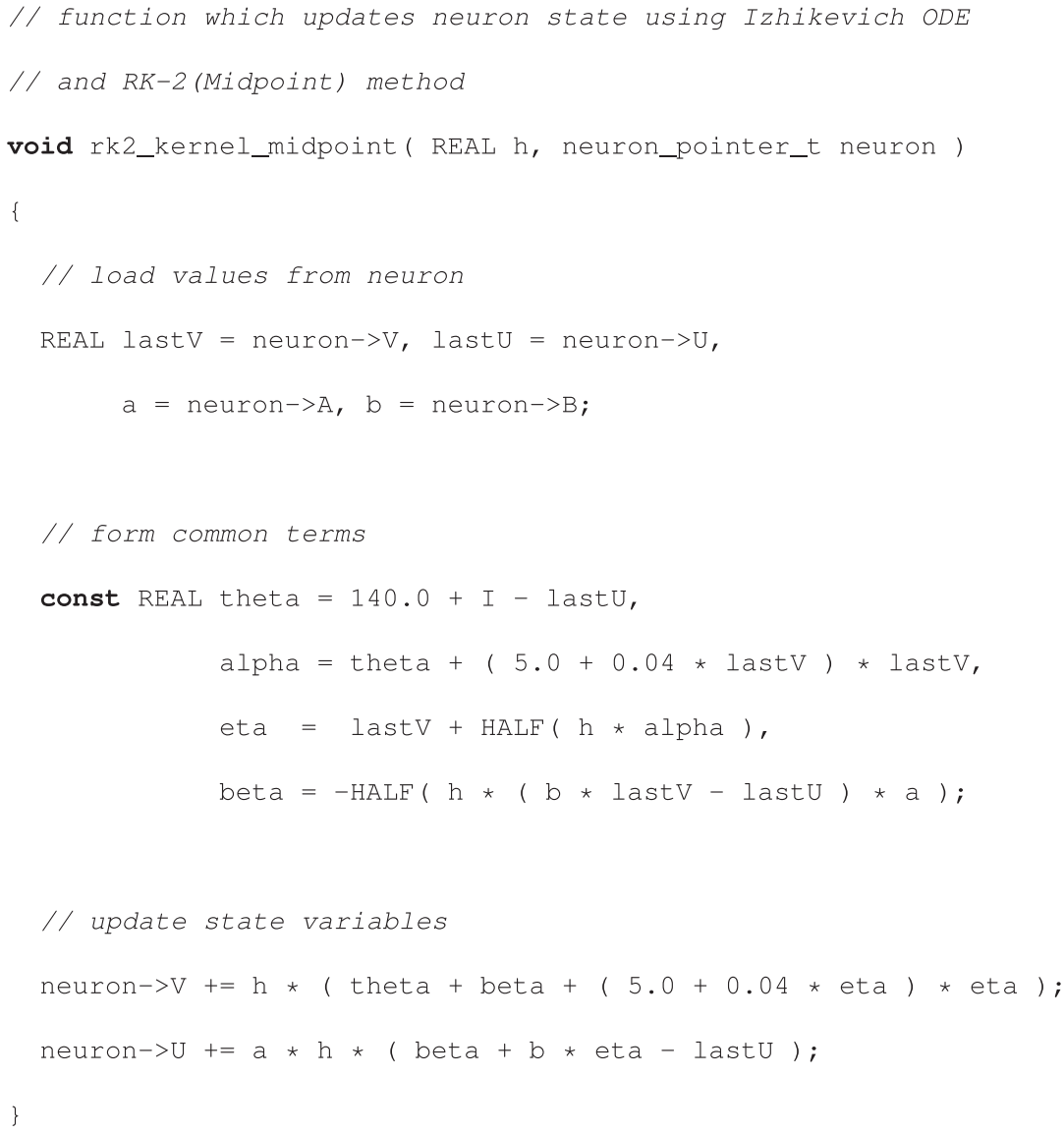



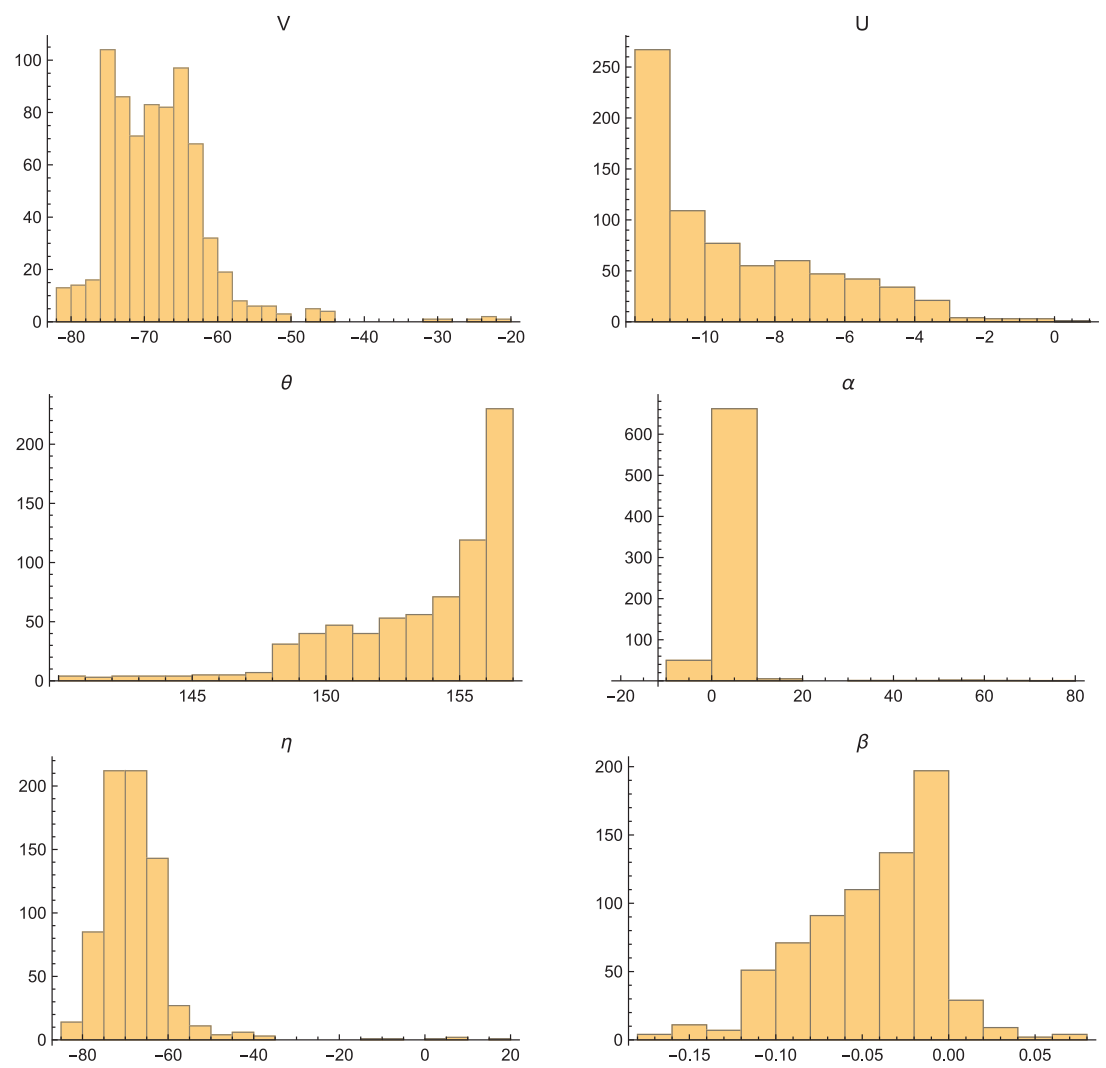

Figure 13: Histograms from sample of state and computational interim variables for RK-2 (Midpoint)-ESR-TQ3-double over 2 seconds of simulation using $1 \mathrm{~ms}$ time step, DC input and TQ3 adjustment (other setups produce similar results).

To indicate typical values used by the state and interim variables in this form, the histograms in Figure 13 show samples from 2 seconds of simulation of the reference neuron using $1 \mathrm{~ms}$ time step with the DC input and TQ3.

C code that implements the core of several of the ESR solvers described in the letter is available at http://www.mitpressjournals.org/doi/suppl /10.1162/NECO_a_00772.

\section{Acknowledgments}

We thank Dave Lester and Jamie Knight for discussions about fixed-point issues and static inline function optimisations; Sergio Davies, Luis Plana, 
Steve Temple, and Simon Davidson for their initial guidance through the SpiNNaker architecture and development environment for M.H.; Jim Garside for looking at assembly output to identify optimization opportunities; Patrick Camilleri for help with LaTeX; and the reviewers for helpful comments and references.

The research leading to these results has received funding from EPSRC (the U.K. Engineering and Physical Sciences Research Council grants EP/G015740/1 and EP/G015775/1), ARM Ltd, the European Research Council under the European Unions Seventh Framework Programme (FP7/2007-2013) / ERC grant agreement 320689 and the EU Flagship Human Brain Project (FP7-604102).

\section{References}

Alsaker, C. A. (2009). Solving systems of differential equations using the Taylor series. (Tech. Rep.). South Dakota School of Mines and Technology.

Babuška, I., Práger, M., \& Vitásek, E. (1966). Numerical processes in differential equations. New York: SNTL/Wiley Interscience.

Bashforth, F., \& Adams, J. C. (1883). An attempt to test the theories of capillary action by comparing the theoretical and measured forms of drops of fluid with an explanation of the method of integration employed in construction the tables which give the theoretical forms of such drops. Cambridge: Cambridge University Press.

Brette, R., \& Guigon, E. (2003). Reliability of spike timing is a general property of spiking model neurons. Neural Computation, 15, 279-308.

Brette, R., Rudolph, M., Carnevale, T., Hines, M., Beeman, D., Bower, J., Diesmann, M., ... \& Destexhe, A. (2007). Simulation of networks of spiking neurons: A review of tools and strategies. J. Comput. Neurosci., 23, 349-398.

Butcher, J. C. (2003). Numerical methods for ordinary differential equations. New York: Wiley.

Chan, R. P. K., \& Tsai, A. Y. J. (2010). On explicit two-derivative Runge-Kutta methods. Numerical Algorithms, 53, 171-194.

Darulova, E., Kuncak, V., Saha, I., \& Majumdar, R. (2013). Synthesis of fixed-point programs (Tech. Rep.). Lausanne: Ecole Polytechnique Fédérale de Lausanne.

Enright, W. H., Higham, D. J., Owren, B., \& Sharp, P. W. (1995). A survey of the explicit Runge-Kutta method (Tech. Rep. 94-291). Toronto: University of Toronto.

Euler, L. (1913). De integratione aequationum differentialium per approximationem. Opera Omina, ser. 1, 11, 424-434.

Furber, S. B., Galluppi, F., Temple, S., \& Plana, L. A. (2014). The SpiNNaker project. Proceedings of the IEEE, 102, 652-665.

Gao, X., Bayliss, S., \& Constantinides, G. (2013). SOAP: Structural optimization of arithmetic expressions for high-level synthesis. In Proceedings of the International Conference on Field-Programmable Technology (pp. 112-119). Piscataway, NJ: IEEE.

Gear, C. W. (1971). Numerical initial value problems in ordinary differential equations. Upper Saddle River, NJ: Prentice Hall.

Gill, S. (1951). A process for the step-by-step integration of differential equations in an automatic digital computing machine. Mathematical Proceedings of the Cambridge Philosophical Society, 47(1), 96-108. 
Hall, G., \& Watt, J. M. (Eds.) (1976). Modern numerical methods for ordinary differential equations. Oxford: Clarendon Press.

Hansel, D., Mato, G., Meunier, C., \& Neltner, L. (1998). On numerical simulations of integrate-and-fire neural networks. Neural Computation, 10, 467-483.

Henrici, P. (1962). Discrete variable methods in ordinary differential equations. New York: Wiley.

Heun, K. (1900). Neue Methoden zur approximativen Integration der Differentialgleichungen einer unabhängigen Veränderlichen. Z. Math. Phys., 45, $23-38$.

Higham, N. J. (1996). Accuracy and stability of numerical algorithms. Philadelphia: SIAM.

Humphries, M. D., \& Gurney, K. (2007). Solution methods for a new class of simple model neurons. Neural Computation, 19, 3216-3225.

ISO/IEC. (2008). TR18037 Programming languages - C - Extensions to support embedded processors. (Tech. Rep.). Geneva: International Organization for Standardization.

Izhikevich, E. M. (2003). Simple model of spiking neurons. IEEE Trans. on Neural Networks, 14, 1569-1572.

Jin, X., Furber, S. B., \& Woods, J. V. (2008). Efficient modelling of spiking neural networks on a scalable chip multiprocessor. In Proceedings of the IJCNN 2008 (pp. 2812-2819). New York: Springer.

Kahan, W. (1965). Further remarks on reducing truncation errors. Comm. ACM, 8, 40.

Kutta, M. W. (1901). Beitrag zur näherungsweisen Integration totaler Differentialgleichungen. Z. Math. Phys., 46, 435-453.

Lambert, J. D. (1973). Computational methods in ordinary differential equations. New York: Wiley.

Lambert, J. D. (1991). Numerical methods for ordinary differential systems: The initial value problem. New York: Wiley.

Mainen, Z. F., \& Sejnowski, T. J. (1995). Reliability of spike timing in neocortical neurons. Science, 268(5216), 1503-1506.

Martel, M. (2009). Enhancing the implementation of mathematical formulas for fixedpoint and floating-point arithmetics. Formal Methods in System Design, 35, 265-278.

Mitsui, T. (1982). Runge-Kutta type integration formulas including the evaluation of the second derivative: Part 1. Publ. RIMS, Kyoto Univ., 18, 325-365.

Ono, H., \& Yoshida, T. (2004). Two-stage explicit Runge-Kutta type methods using derivatives. Japan J. Indust. Appl. Math., 21, 361-374.

Press, W. H., Teukolsky, S. A., Vetterling, W. T., \& Flannery, B. P. (1992). Numerical recipes in $\mathrm{C}$ (2nd ed.). Cambridge: Cambridge University Press.

Runge, C.D.T. (1895). Über die numerische Auflösung von Differentialgleichungen. Math. Ann., 46, 167-178.

Shintani, H. (1972). On explicit one-step methods utilizing the second derivative. Hirsohima Math. J., 2, 353-368.

Stewart, R. D., \& Bair, W. (2009). Spiking neural network simulation: Numerical integration with the Parker-Sochacki method. J. Comput. Neurosci., 27, 115-133.

Stimberg, M., Goodman, D. F. M., Benichoux, V., \& Brette, R. (2014). Equationoriented specification of neural models for simulations. Frontiers in Neuroinformatics, 8(6).

Touboul, J. (2009). Importance of the cutoff value in the quadratic adaptive integrateand-fire model. Neural Computation, 21, 2114-2122. 
Touboul, J. (2010). On the simulation of nonlinear bidimensional spiking neuron models. Neural Computation, 23, 1704-1742.

Vitásek, E. (1969). The numerical stability in solution of differential equations. Conf. on Numerical Solution of Differential Equations, 109, 87-111.

Wolfram Research (2014). Mathematica (version 10.0 ed.). Champaign, IL: Wolfram Research.

Received October 31, 2014; accepted June 4, 2015. 